\title{
On 1-Harmonic Functions ${ }^{\star}$
}

\author{
Shihshu Walter WEI
}

Department of Mathematics, The University of Oklahoma, Norman, Ok 73019-0315, USA

E-mail:wwei@ou.edu

Received September 18, 2007, in final form December 17, 2007; Published online December 27, 2007

Original article is available at http://www.emis.de/journals/SIGMA/2007/127/

\begin{abstract}
Characterizations of entire subsolutions for the 1-harmonic equation of a constant 1-tension field are given with applications in geometry via transformation group theory. In particular, we prove that every level hypersurface of such a subsolution is calibrated and hence is area-minimizing over $\mathbb{R}$; and every 7-dimensional $S O(2) \times S O(6)$-invariant absolutely area-minimizing integral current in $\mathbb{R}^{8}$ is real analytic. The assumption on the $S O(2) \times S O(6)$-invariance cannot be removed, due to the first counter-example in $\mathbb{R}^{8}$, proved by Bombieri, De Girogi and Giusti.
\end{abstract}

Key words: 1-harmonic function; 1-tension field; absolutely area-minimizing integral current

2000 Mathematics Subject Classification: 53C40; 53C42

\section{Introduction}

The study of 1-harmonic functions, or more generally that of $p$-harmonic maps is an area of an active research that is related with many branches of mathematics. For instance, in a celebrated paper of Bombieri, De Girogi and Giusti [3], a 1-harmonic function has been constructed to provide a counter-example for interior regularity of the solution to the co-dimension one Plateau problem in $\mathbb{R}^{n}$ for $n>7$. Recall a $C^{1}$ functions $f: \mathbb{R}^{n} \rightarrow \mathbb{R}$ is said to be 1-harmonic if it is a weak solution of 1-harmonic equation

$$
\operatorname{div}\left(\frac{\nabla f}{|\nabla f|}\right)=0
$$

where $|\nabla f|$ is the length of the gradient $\nabla f$ of $f$, and for a $C^{2}$ function $f$ without a critical point, $\operatorname{div}\left(\frac{\nabla f}{|\nabla f|}\right)$ is said to be the 1-tension field of $f$.

In this paper, characterizations of entire subsolutions for the 1-harmonic equation of a constant 1-tension field are given in various aspects, and their relationships with calibration geometry are established (cf. Theorem 2, Corollary 3). As applications, we prove via transformation group theory (cf. $[9,10,13,2,21]$ ) that the cone over $S^{1} \times S^{5}$ is not minimizing in $\mathbb{R}^{8}$ but is stable; that any 7 -dimensional $S O(2) \times S O(6)$-invariant absolutely area-minimizing integral current in $\mathbb{R}^{8}$ is real analytic; and that the only 7 -dimensional $S O(3) \times S O(5)$-invariant minimizing integral current with singularities in $\mathbb{R}^{8}$ is the cone over $S^{2} \times S^{4}$, and is minimizing over $\mathbb{R}$ (cf. Theorems $3-5)$. These results improved an early partial proof by numerical computation done by Plinio Simoes [17] in his Berkeley thesis. The assumption on the $S O(2) \times S O(6)$-invariance cannot be removed, due to the first counter-example of Bombieri, De Girogi and Giusti that the cone over $S^{3}\left(\frac{1}{\sqrt{2}}\right) \times S^{3}\left(\frac{1}{\sqrt{2}}\right) \subset S^{7}(1)$ is area-minimizing in $\mathbb{R}^{8}$. It should be pointed out that Fang-Hua Lin [14] proved that the cone over $S^{1} \times S^{5}$ is one-sided area-minimizing and is stable by a different method. By constructing 1-harmonic functions on hyperbolic space $H^{n}, H^{n} \times H^{n}$,

\footnotetext{
${ }^{\star}$ This paper is a contribution to the Proceedings of the 2007 Midwest Geometry Conference in honor of Thomas P. Branson. The full collection is available at http://www.emis.de/journals/SIGMA/MGC2007.html
} 
$H^{n} \times S O(n, 1)$ and many other associated spaces, S.P. Wang and the author [19] show the Bernstein Conjecture in these spaces to be false in all dimensions. In particular, these constructions give the first set of examples of complete, smooth, embedded, minimal (hyper-)surfaces in hyperbolic space $H^{n}$ in all dimensions (cf. also Remark 3(ii)).

\section{Fundamentals in geometric measure theory}

For our subsequent development, we recall some fundamental facts, definitions, and notations, for which the reference is Federer's book [5] and paper [7].

Let $N$ denote an $n$-dimensional Riemannian manifold and denote by $\mathcal{R}_{p}^{\text {loc }}(N)$ the set of $p$ dimensional, locally rectifiable currents (of Federer and Fleming, cf. [8]) on $N$. For $S \in \mathcal{R}_{p}^{\text {loc }}(N)$, denote the mass of $S$ by $\mathbf{M}(S)$, and the boundary of $S$ by $\partial S$, and is given by $(\partial S)(w)=S(d w)$, where $w$ is a smooth $p$-form and $d$ is the exterior differentiation. From a calculus of variational viewpoint, we make the following

Definition 1. A current $T \in \mathcal{R}_{k}^{\text {loc }}(N)$ is said to be stationary if $\left.\frac{d}{d t} \mathbf{M}\left(\phi_{t_{*}}^{V}(T)\right)\right|_{t=0}$ for all vector fields $V$ on $N$ with compact support where $\phi_{t}^{V}$ is the flow associated with $V$, and stable if for every vector fields $V$ on $N$ with compact support, there exists an $\epsilon>0$ such that $\mathbf{M}(T) \leq$ $\mathbf{M}\left(\phi_{t_{*}}^{V}(T)\right)$ for $|t|<\epsilon$.

We are primarily interested in minimizing currents.

Definition 2. A current $T \in \mathcal{R}_{k}^{\text {loc }}(N)$ is homologically (resp. absolutely) area-minimizing over $\mathbb{Z}$ if for all compact sets $K \subset M$, we have $\mathbf{M}\left(\phi_{K} T\right) \leq \mathbf{M}\left(\left(\phi_{K} T\right)+S\right)$ for all $S \in \mathcal{R}_{k}^{\text {loc }}(N)$ having compact support and being the boundary of some current in $\mathcal{R}_{k+1}^{\text {loc }}(N)$ with compact support (resp. the empty boundary)(here $\phi_{K}$ denotes the characteristic function on $K$ ).

Using a dimension reduction technique, Federer proves that the support of an area-minimizing integral current $T$ [8] minus another compact set $\mathrm{S}$ whose Hausdorff dimension does not exceed $n-8$ is an $(n-1)$-dimensional analytic manifold [6]. Hence, if $n \leq 7$, then $\mathrm{S}=\varnothing$. If $n=8$, $\mathrm{S}$ consists of at most isolated points $[5,5.4 .16]$. This result is optimal by the counter-example due to Bombieri-De Giorgi-Giusti [3] that $\left\{x \in \mathbb{R}^{2 m}: x_{1}^{2}+\cdots+x_{m}^{2}=x_{m+1}^{2}+\cdots+x_{2 m}^{2}\right\}$ is an area-minimizing cone over the product of $(m-1)$-spheres $\left\{x \in \mathbb{R}^{2 m}: x_{1}^{2}+\cdots+x_{m}^{2}=x_{m+1}^{2}+\cdots\right.$ $\left.+x_{2 m}^{2}=\frac{1}{2}\right\}$ in $\mathbb{R}^{2 m}$ for $m \geq 4$.

The union of the groups $\mathcal{F}_{m, K}(U)=\left\{R+\partial T: R \in \mathcal{R}_{m, K}(U), T \in \mathcal{R}_{m+1, K}(U)\right\}$ corresponding to all compact $K \subset U$ is the group $\mathcal{F}_{m}(U)$ of $m$-dimensional integral flat chains in an open subset $U$ of $\mathbb{R}^{n}$. We denote the group of $m$-dimensional integral flat chains, cycles and boundaries by $\mathcal{F}_{m}(A)=\mathcal{F}_{m}\left(\mathbb{R}^{n}\right) \cap\{S:$ spt $S \subset A\}, \mathcal{Z}_{m}(A, B)=\mathcal{F}_{m}(A) \cap\left\{S: \partial S \subset \mathcal{F}_{m}(B)\right.$ or $\left.m=0\right\}$, and $\mathcal{B}_{m}(A, B)=\left\{R+\partial T: R \in \mathcal{F}_{m}(B), T \in \mathcal{F}_{m+1}(A)\right\}$ respectively. Similarly, we define and denote $\mathbf{F}_{m}(A), \mathbf{Z}_{m}(A, B)$ and $\mathbf{B}_{m}(A, B)$ the vector space of $m$-dimensional real flat chains, cycles and boundaries respectively, where $B \subset A$ are compact Lipschitz neighborhood retract in $U$.

For every positive convex parametric integrand $\psi$, and every compact subset $K$ of $A$, we define $\mathcal{Z}_{m, K}(A, B)=\mathcal{Z}_{m}(A, B) \cap\{R: \operatorname{spt} R \subset K\}, \mathcal{B}_{m, K}(A, B)=\mathcal{B}_{m}(A, B) \cap\{R:$ spt $R \subset K\}$, $\mathbf{Z}_{m, K}(A, B)=\mathbf{Z}_{m}(A, B) \cap\{R: \operatorname{spt} R \subset K\}$, and $\mathbf{B}_{m, K}(A, B)=\mathbf{B}_{m}(A, B) \cap\{R: \operatorname{spt} R \subset K\}$, and make the following

Definition 3. An $m$-dimensional rectifiable current $Q$ (resp. $Q^{\prime}$ ) is said to be absolutely (resp. homologically) $\psi$-minimizing in $K$ with respect to $(A, B)$ over $\mathbb{Z}$ if

$$
\begin{aligned}
& \int_{Q} \psi=\inf \left\{\int_{S} \psi: S \in \mathcal{F}_{m, K}(U), Q-S \in \mathcal{Z}_{m, K}(A, B)\right\} \\
& \text { (resp. } \left.\int_{Q^{\prime}} \psi=\inf \left\{\int_{S} \psi: S \in \mathcal{B}_{m, K}(U), Q^{\prime}-S \in \mathcal{B}_{m, K}(A, B)\right\}\right) .
\end{aligned}
$$


Definition 4. An $m$-dimensional real flat chain $Q$ (resp. $Q^{\prime}$ ) is said to be absolutely (resp. homologically) $\psi$-minimizing in $K$ with respect to $(A, B)$ over $\mathbb{R}$ if

$$
\begin{aligned}
& \int_{Q} \psi=\inf \left\{\int_{S} \psi: S \in \mathbf{F}_{m, K}(U), Q-S \in \mathbf{Z}_{m, K}(A, B)\right\} \\
& \left(\text { resp. } \int_{Q^{\prime}} \psi=\inf \left\{\int_{S} \psi: S \in \mathbf{B}_{m, K}(U), Q^{\prime}-S \in \mathbf{B}_{m, K}(A, B)\right\}\right) .
\end{aligned}
$$

We will make comparisons between real and integral absolute (resp. homological) minimizing currents in the subsequent Sections 3, 4, and 5.

\section{Characterizations of subsolutions for 1-harmonic equation of constant 1-tension field}

We connect an entire subsolution of this sort, with a calibration. Recall a calibration is a closed form with comass 1 .

Lemma 1. Let $M$ be a complete noncompact Riemannian manifold. For any $x_{0} \in M$ and any pair of positive numbers $s, t$ with $s<t$, there exists a rotationally symmetric Lipschitz continuous function $\psi(x)=\psi(x ; s, t)$ and a constant $C_{1}>0$ (independent of $x_{0}, s, t$ ) with the properties:

$$
\begin{aligned}
& \text { (i) } \psi \equiv 1 \text { on } B\left(x_{0} ; s\right) \text {, and } \psi \equiv 0 \text { off } B\left(x_{0} ; t\right) \\
& \text { (ii) }|\nabla \psi| \leq \frac{C_{1}}{t-s} \text {, a.e. on } M \text {. }
\end{aligned}
$$

Proof. (cf. Andreotti and Vesentini [1], Yau [22], Karp [11]).

Theorem 1. Let $\Omega$ be a domain in $\mathbb{R}^{n}$ containing a ball $B\left(x_{0}, r\right)$ of radius $r$, centered at $x_{0}$, and $g: \Omega \rightarrow \mathbb{R}$ be a continuous function with $g \geq 0$, and $c=\inf _{x \in B\left(x_{0}, \frac{r}{2}\right)} g(x)$. Let $f: \Omega \rightarrow \mathbb{R}$ be a $C^{1}$ weak solution of

$$
\operatorname{div}\left(\frac{\nabla f}{|\nabla f|}\right)=g(x) \quad \text { on } \Omega,
$$

then the inf imum c satisfies

$$
0 \leq c \leq \frac{C_{1} 2^{n}}{r}
$$

where $C_{1}$ is as in (3.1).

Proof. Let $\psi \geq 0$ be as in Lemma 1 , in which $M=\mathbb{R}^{n}, t=r, s=\frac{r}{2}$. Choose $\psi$ to be a test function in the distribution sense of (3.2). Then via the assumption on $g$, and Cauchy-Schwarz inequality we have:

$$
\begin{aligned}
\int_{B\left(x_{0}, \frac{r}{2}\right)} c \psi(x) d x & \leq \int_{B\left(x_{0}, \frac{r}{2}\right)} g(x) \psi(x) d x \\
& \leq \int_{B\left(x_{0}, r\right)} g(x) \psi(x) d x=-\int_{B\left(x_{0}, r\right)} \frac{\nabla f}{|\nabla f|} \cdot \nabla \psi d x \leq \int_{B\left(x_{0}, r\right)}|\nabla \psi| d x .
\end{aligned}
$$

Hence,

$$
c \operatorname{Vol}\left(B\left(x_{0}, \frac{r}{2}\right)\right) \leq \frac{C_{1}}{r} \operatorname{Vol}\left(B\left(x_{0}, r\right)\right)
$$

yields the desired. 
Corollary 1. Let $f: \mathbb{R}^{n} \rightarrow \mathbb{R}$ be a $C^{1}$ weak subsolution of 1-harmonic equation (1.1) with constant 1-tension field c, i.e. $0 \leq \operatorname{div}\left(\frac{\nabla f}{|\nabla f|}\right)=c$ in the distribution sense. Then $f$ is a 1 -harmonic function.

Corollary 2. There does not exist a $C^{1}$ weak subsolution $f: \mathbb{R}^{n} \rightarrow \mathbb{R}$ of equation (3.2) with $\lim _{r \rightarrow \infty} \inf _{x \in B\left(x_{0}, r\right)} g(x)>0$, for any $x_{0} \in \mathbb{R}^{n}$.

Let $A \subset R^{n}$ be an open set. We denote $B V_{\text {loc }}(A)=\left\{f \in L_{\text {loc }}^{1}(A)\right.$ : the distributional derivatives $D_{i} f$ of $f$ are (locally) measures $\}=\left\{f \in L_{\text {loc }}^{1}(A): \operatorname{supp} \phi_{n} \subset K \subset A, \phi_{n} \rightarrow 0\right.$ uniformly, imply $\left.\left(\frac{\partial}{\partial x_{i}} f\right) \phi_{n} \rightarrow 0\right\}$. Let $D f=\left(D_{1} f, \ldots, D_{n} f\right)$ denote the gradient of $f$ in the sense of distributions and $|D f|$ the scalar measure defined by $\int_{K}|D f|=\sup \int_{K} \sum_{i} \epsilon_{i}(x) D_{i} f$, where the supremum is taken over all sets $\left\{\epsilon_{i}(x), i=1, \ldots, n\right\}$ of $C^{\infty}(K)$ functions which satisfy $\sum \epsilon_{i}^{2}(x) \leq 1$.

Definition 5. A function $f \in B V_{\text {loc }}(A)$ has least gradient in $A$ if for every $g \in B V_{\text {loc }}(A)$, with compact support $K \subset A$ we have

$$
\int_{K}|D f| \leq \int_{K}|D(f+g)|
$$

Definition 6. Let $E$ be a set in $\mathbb{R}^{n}$ and $\phi_{E}$ its characteristic function. $E$ has an oriented boundary of least area with respect to $A$, if $(i) \phi_{E} \in B V_{\text {loc }}(A)$ and (ii) for each $g \in B V_{\text {loc }}(A)$ with compact support $K \subset A$ we have $\int_{K}\left|D \phi_{E}\right| \leq \int_{K}\left|D\left(\phi_{E}+g\right)\right|$.

Theorem 2. Let $f \in H_{\mathrm{loc}}^{1,1}\left(\mathbb{R}^{n}\right)$, and $\nabla f(x) \neq 0$ for every $x$ in $\mathbb{R}^{n}$. Let $E_{\lambda}=\{x: f(x) \geq \lambda\}$, and $S_{\lambda}=\{x: f(x)=\lambda\}$. We denote the set of integers by $\mathbb{Z}$. Then the following thirteen statements (1)-(13) are equivalent and each of them implies the fourteenth statement (14).

1. $f: \mathbb{R}^{n} \rightarrow \mathbb{R}$ is a $C^{1}$ weak subsolution of (1.1) with constant 1-tension field.

2. $f$ is a $C^{1}$ weak solution of (1.1) on $\mathbb{R}^{n}$.

3. $f$ is a $C^{1} 1$-harmonic function on $\mathbb{R}^{n}$.

4. For each $\left(a, t_{0}\right)=\left(a_{1}, \ldots, a_{n-1}, t_{0}\right) \in S_{\lambda}$, there exists a neighborhood $\mathcal{D}$ of a in $\mathbb{R}^{n-1}$, and a unique real analytic function $\eta: \mathcal{D} \rightarrow \mathbb{R}$ such that $\eta(a)=t_{0}, f\left(x_{1}, \ldots, x_{n-1}, \eta\left(x_{1}, \ldots\right.\right.$, $\left.\left.x_{n-1}\right)\right)=\lambda$ and $\operatorname{div}\left(\frac{\nabla \eta}{\sqrt{1+|\nabla \eta|^{2}}}\right)=0$ on $\mathcal{D}$.

5. Each level hypersurface $S_{\lambda}$ is minimal in $\mathbb{R}^{n}$.

6. $\frac{* d f}{|d f|}$ is a globally defined "weakly" closed form with comass 1 .

7. $f$ is a function of least gradient in $\mathbb{R}^{n}$.

8. Each $E_{\lambda}, \lambda \in \mathbb{R}$ has an oriented boundary of least area with respect to $\mathbb{R}^{n}$.

9. Each level hypersurface $S_{\lambda}$ is absolutely area-minimizing in $\mathbb{R}^{n}$ over $\mathbb{Z}$.

10. Each level hypersurface $S_{\lambda}$ is absolutely area-minimizing in $\mathbb{R}^{n}$ over $\mathbb{R}$.

11. Each level hypersurface $S_{\lambda}$ is homologically area-minimizing in $\mathbb{R}^{n}$ over $\mathbb{R}$.

12. Each level hypersurface $S_{\lambda}$ is homologically area-minimizing in $\mathbb{R}^{n}$ over $\mathbb{Z}$.

13. Each level hypersurface $S_{\lambda}$ is stable in $\mathbb{R}^{n}$.

14. If $f \in C^{2}\left(\mathbb{R}^{n}\right)$, then $\frac{* d f}{|d f|}$ is closed and the restriction $\left.\frac{* d f}{|d f|}\right|_{S_{\lambda}}$ is its volume form, hence each $S_{\lambda}$ is real absolutely area-minimizing in $\mathbb{R}^{n}$ over $\mathbb{R}$. 
Corollary 3. Every level hypersurface of a $C^{2}$ subsolution of 1 -harmonic equation on $\mathbb{R}^{n+1}$ with constant 1-tension field is calibrated and hence is area-minimizing over $\mathbb{R}$.

Proof. $(1) \Leftrightarrow(2) \Leftrightarrow(3)$ : This follows immediately from Corollary 1 .

$(2) \Leftrightarrow(4):(\Rightarrow)$ Let $f\left(x_{1}, \ldots, x_{n-1}, t\right)=\eta\left(x_{1}, \ldots, x_{n-1}\right)-t$. The assertion follows from the implicit function theorem and

$$
0=\int \frac{\sum_{i=1}^{n-1} \frac{\partial f}{\partial x_{i}} \frac{\partial \varphi}{\partial x_{i}}}{|\nabla f|}+\int \frac{\frac{\partial f}{\partial t}}{|\nabla f|} \frac{\partial \varphi}{\partial t}=\int \sum_{i=1}^{n-1} \frac{\frac{\partial \eta}{\partial x_{i}}}{\sqrt{1+|\nabla \eta|^{2}}} \frac{\partial \varphi}{\partial x_{i}}
$$

for all $\varphi \in C_{0}^{\infty}(\mathcal{D} \times \mathbb{R})$. The regularity of solutions of minimal surface equation implies that $\eta$ is real analytic and completes the proof. $(\Leftarrow)$ This follows immediately from (3.3).

$(4) \Leftrightarrow(5)$ : This is due to the fact that the graph of a solution to the minimal surface equation on $\mathcal{D}$ is a minimal hypersurface in $\mathcal{D} \times \mathbb{R}$.

$(2) \Leftrightarrow(6)$ : This follows from the following: For every $\phi \in C_{0}^{\infty}(A)$,

$$
\begin{aligned}
\int_{A} \frac{* d f}{|d f|} \wedge d \phi & =\int_{A} \sum_{i, j=1}^{n}(-1)^{i-1} \frac{\frac{\partial f}{\partial x_{i}}}{|\nabla f|} d x^{1} \wedge \cdots \wedge d x^{i-1} \wedge d x^{i+1} \wedge \cdots \wedge d x^{n} \wedge \frac{\partial \phi}{\partial x_{j}} d x^{j} \\
& =\int_{A} \sum_{i=1}^{n}(-1)^{n-1} \frac{\frac{\partial f}{\partial x_{i}} \frac{\partial \phi}{\partial x_{i}}}{|\nabla f|} d x^{1} \wedge \cdots \wedge d x^{i} \wedge \cdots \wedge d x^{n} .
\end{aligned}
$$

$(2) \Rightarrow(7)$ : let us first assume that $g \in C_{0}^{1}(A)$. Let $h(t)=\int|D(f+t g)|$. Then

$$
h^{\prime}(t)=\int \frac{\left(\sum_{i=1}^{n} \frac{\partial(f+t g)}{\partial x_{i}} \frac{\partial g}{\partial x_{i}}\right)}{\left(\sum_{i=1}^{n}\left(\frac{\partial(f+t g)}{\partial x_{i}}\right)^{2}\right)^{\frac{1}{2}}} .
$$

Hence $h^{\prime}(0)=0$ by assumption. Furthermore,

$$
h^{\prime \prime}(t)=\int \frac{\left(\sum_{i=1}^{n}\left(\frac{\partial g}{\partial x_{i}}\right)^{2}\right)\left(\sum_{i=1}^{n}\left(\frac{\partial(f+t g)}{\partial x_{i}}\right)^{2}\right)-\left(\sum_{i=1}^{n} \frac{\partial(f+t g)}{\partial x_{i}} \frac{\partial g}{\partial x_{i}}\right)^{2}}{\left[\sum_{i=1}^{n}\left(\frac{\partial(f+t g)}{\partial x_{i}}\right)^{2}\right]^{\frac{3}{2}}} \geq 0,
$$

by the Cauchy-Schwarz inequality. Therefore $\int|D f|=h(0) \leq h(1)=\int|D(f+g)|$. If $g \in$ $B V_{\text {loc }}(A)$ with compact support $K$ and let $D g=G_{1}+G_{2}$ where $G_{1}$ is completely continuous and $G_{2}$ is the singular part of $D g$ with support $N_{g}$ of measure zero. Then we have $\int_{K} \mid D(f+$ $g)\left|=\int_{K}\right| D f+G_{1}\left|+\int_{K}\right| G_{2} \mid$ because $f \in H_{\text {loc }}^{1,1}(A)$. Let $g_{\varepsilon}=g * \psi_{\varepsilon}$ where $\psi_{\varepsilon}$ is a mollifier. Then $g \in C_{0}^{1}(A)$ and $\int_{K_{\epsilon}}|D f| \leq \int_{K_{\epsilon}}\left|D\left(f+g_{\epsilon}\right)\right| \leq \int_{K_{\epsilon}}\left|D f+G_{1} * \Psi_{\epsilon}\right|+\int_{A}\left|G_{2} * \Psi_{\epsilon}\right|$, where $K_{\epsilon}=\{x \in A: \operatorname{dist}(x, K)<\epsilon\}$. Letting $\epsilon \rightarrow 0$ completes the proof (cf. [3]).

$(7) \Rightarrow(8)$ : This follows from Coarea formula for BV functions [15], $\int_{K}|D f|=\int_{-\infty}^{\infty}\left(\int_{K}\left|D \phi_{\lambda}\right|\right) d \lambda$ together with two observations:

(i) If $f_{1}$ and $f_{2}$ satisfy (3.3), so does $\sup \left(f_{1}, f_{2}\right)$.

(ii) If $f_{i} \in B V_{\text {loc }}(A), f_{i} \rightarrow f$ in $L_{\text {loc }}^{1}$ and each $f_{i}$ satisfies (3.3), so does also $f \in B V_{\text {loc }}(A)$ and satisfies (3.3).

For detailed proof see [16]. 
$(8) \Rightarrow(9)$ : Let $\phi_{\lambda}=\phi_{E_{\lambda}}$. Since for every $x$ in $\mathbb{R}^{n}, \nabla f(x) \neq 0, \partial E_{\lambda}=S_{\lambda}$ for $S_{\lambda} \neq \varnothing$. It follows from a theorem of Miranda [15] that on any compact set $K$ in $\mathbb{R}^{n}$, the Hausdorff $(n-1)$-measure

$$
\mathcal{H}^{n-1}\left(K \cap S_{\lambda}\right)=\int_{K}\left|D \phi_{\lambda}\right| \leq \int_{K}\left|D\left(\phi_{\lambda}+g\right)\right|=\mathcal{H}^{n-1}(K \cap T)
$$

for all sets $T$ with $\partial(K \cap T)=\partial\left(K \cap S_{\lambda}\right)$.

$(9) \Rightarrow(10):$ It follows from Theorem 6 .

$(10) \Rightarrow(11) \Rightarrow(12)$ : Since absolute area-minimization over $\mathbb{R} \Rightarrow$ homological area-minimization over $\mathbb{R} \Rightarrow$ homological area-minimization over $\mathbb{Z}$.

$(12) \Rightarrow(13) \Rightarrow(5)$ : Since homological minimization over $\mathbb{Z} \Rightarrow$ stability $\Rightarrow$ minimality. This completes the proof of $(1) \Leftrightarrow \cdots \Leftrightarrow(13)$.

$(2) \Rightarrow(14)$ : If $f \in C^{2}(A)$ then by $(3.4) \frac{* d f}{|d f|}$ is closed. Now let $e_{1}, \ldots, e_{n-1}$ be an orthonormal basis for the tangent space of $S_{\lambda}$ at $x_{0}$ and $\nu$ a unit normal vector at $x_{0}$. We denote by tilde " " the canonical isomorphism between a tangent space and its dual space. To show $\frac{* d f}{|d f|}$ has comass 1 , note for any $(n-1)$-vector field $\xi$,

$$
\begin{aligned}
\frac{* d f}{|d f|}(\xi) & =\left(* \frac{\widetilde{\nabla f}}{|\nabla f|}\right)(\xi) \quad\left(\text { because } \frac{d f}{|d f|}(X)=\frac{X f}{|\nabla f|}=\left\langle\frac{\nabla f}{|\nabla f|}, X\right\rangle\right) \\
& =(* \tilde{\nu})(\xi)=\left(e_{1} \wedge \widetilde{\cdots \wedge} e_{n-1}\right)(\xi)=\left\langle e_{1} \wedge \cdots \wedge e_{n-1}, \xi\right\rangle .
\end{aligned}
$$

In particular $\frac{* d f}{|d f|}\left(e_{1} \wedge \cdots \wedge e_{n-1}\right)=1, \frac{* d f}{|d f|}(\xi) \leq 1$ and $\left.\frac{* d f}{|d f|}\right|_{S_{\lambda}}=$ volume element of $S_{\lambda}$. By the formalism of Stokes theorem, for any integral current $T$ with $\partial T=\partial\left(S_{\lambda} \cap B_{r}\right)$

$$
\begin{aligned}
M\left(S_{\lambda} \cap B_{r}\right) & =\left(S_{\lambda} \cap B_{r}\right)\left(\frac{* d f}{|d f|}\right)=T\left(\frac{* d f}{|d f|}\right) \\
& =\int \frac{* d f}{|d f|}\left(\overrightarrow{T_{x}}\right) d\|T\|(x) \leq \int d\|T\|=M(T),
\end{aligned}
$$

where $\vec{T}$ is the field of oriented unit tangent planes to $T$.

Remark 1. In Theorem 2, if one replace $\mathbb{R}^{n}$ with an open subset $A$ in $\mathbb{R}^{n}$, then assertions $(2) \Leftrightarrow \cdots \Leftrightarrow(13) \Rightarrow(14)$ remain to be true.

Remark 2. Concerning the assertion $(2) \Rightarrow(7)$, a stronger theorem can be found in [3]: Let $A \subset R^{n}$ be an open set and let $f \in H_{\text {loc }}^{1,1}(A)$. Suppose that $(i) \mathcal{H}_{n}(\{x \in A:|\nabla f|=0\})=0$, (ii) $\mathcal{H}_{n-1}(N)=0$ where $N$ is a closet set in $A$, (iii) $\int_{A-N}|\nabla f|^{-1} \sum_{i=1}^{n} \frac{\partial f}{\partial x_{i}} \frac{\partial \phi}{\partial x_{i}} d x=0$ for every $\phi \in C_{0}^{1}(A-N)$. Then $f$ has least gradient with respect to $A$.

Remark 3. (i) The assertion (7) $\Rightarrow(9)$ is due to Miranda.

(ii) Connecting the assertions (5), (6), and (12) on Riemannian manifolds, S.P. Wang and the author [19] prove that if each level hypersurface of a smooth function $f: M \rightarrow \mathbb{R}$ on an oriented Riemannian manifold $M$ with nowhere vanishing $\nabla f$, is minimal, then there exists a closed form with comass 1 on $M$ and hence each level hypersurface is homologically area-minimizing over $\mathbb{R}$.

Corollary 4. Let $A$ be an open subset in $\mathbb{R}^{n}, N$ be a closed subset in $A$ with $\mathcal{H}_{n-1}(N)=0$. Then the graph of any weak solution of the minimal surface equation $\sum_{i=1}^{n} \frac{\partial}{\partial x_{i}}\left(\frac{\frac{\partial f}{\partial x_{i}}}{\sqrt{1+|\nabla f|^{2}}}\right)=0$ on $A-N$ is in fact absolutely area-minimizing in $A \times \mathbb{R} \subset \mathbb{R}^{n+1}$ over $\mathbb{R}$. 
Proof. Applying (3.4) in which " $f\left(x_{1}, \ldots, x_{n-1}, t\right)=\eta\left(x_{1}, \ldots, x_{n-1}\right)-t$ " is replaced with " $F\left(x_{1}, \ldots, x_{n}, t\right)=f\left(x_{1}, \ldots, x_{n}\right)-t$ ", and Remark 2 , we have that $F$ is a $C^{1} 1$-harmonic function in $A$. By Theorem 2, the zero level set $S_{0}=\left\{\left(x_{1}, \ldots, x_{n}, t\right): t=f\left(x_{1}, \ldots, x_{n}\right)\right\}$ is absolutely area-minimizing in $A \times \mathbb{R} \subset \mathbb{R}^{n+1}$ over $\mathbb{R}$.

\section{Further applications}

A natural question arises: Are Bombieri-De Giorgi-Giusti and Lawson cones the only $S O(m)$ $\times S O(n)$-invariant singular absolutely area-minimizing integral currents in Euclidean space $\mathbb{R}^{m+n+2}$ ? The answer is affirmative. Combining the theory of 1 -harmonic functions developed, and the techniques of transformation groups in [10, 13, 2], and [21], evolved from the ideas in [9], one obtains the following:

Theorem 3. The cone $C\left(S^{m} \times S^{n}\right)$ over $S^{m} \times S^{n}$ is the unique singular absolutely areaminimizing hypersurface in the class of $S O(m+1) \times S O(n+1)$-invariant integral currents in $\mathbb{R}^{m+n+2}$ over $\mathbb{R}$ for $m+n>7$ or $m+n=6,|m-n| \leq 2$. (It is known that the cone is not even stable otherwise.)

Proof. Assume $m=n$. Let Lie group $G=S O(n+1) \times S O(n+1)$ acting on manifold $\mathbb{R}^{n+1} \times \mathbb{R}^{n+1}$ in the standard way, i.e. assigning $((A, B),(x, y)) \in G \times \mathbb{R}^{2 n+2}$ to $(A \cdot x, B \cdot y) \in$ $\mathbb{R}^{2 n+2}$, where "." is the matrix multiplication. Then the collection $X$ of principle orbits is given by $X=\left\{(x, y) \in \mathbb{R}^{2 n+2}:|x||y| \neq 0\right\}$, where "| $\cdot \mid$ " is the length of "." in $\mathbb{R}^{n+1}$. The orbit space which is stratified, can be represented as $\mathbb{R}^{2 n+2} / G=\left\{(u, v) \in \mathbb{R}^{2}: u, v \geq 0\right\}=$ $X \cup\left\{(u, v) \in \mathbb{R}^{2}: u=0, v>0\right\} \cup\left\{(u, v) \in \mathbb{R}^{2}: u>0, v=0\right\} \cup\{(0,0)\}$. The canonical metric on $\mathbb{R}^{2 n+2} / G$ (compatible with the fibration over each stratum) is the usual flat one $d s_{0}^{2}=d u^{2}+d v^{2}$. The canonical projection $\pi: \mathbb{R}^{2 n+2} \rightarrow \mathbb{R}^{2 n+2} / G$ is given by $\pi(x, y)=(|x|,|y|)$, and let $X / G=\pi(X)$. Then the length of a curve $\sigma$ in $\left(X / G, d s_{0}^{2}\right)$ is the length of any orthogonal trajectory through the corresponding orbits in $X$, and $2 n$-dimensional volume of $\pi^{-1}((u, v))$ (which is diffeomorphic to $S^{n} \times S^{n}$ ) is proportional to $u^{n} v^{n}$, for $(u, v) \in X / G$. Thus if we choose the metric $d s^{2}=u^{2 n} v^{2 n}\left(d u^{2}+d v^{2}\right)$ on $\mathbb{R}^{2 n+2} / G$, then by Fubini's theorem, the length of a curve $\sigma$ in $\left(\mathbb{R}^{2 n+2} / G, d s^{2}\right)$ is equal to $(2 n+1)$-dimensional volume of hypersurface $\pi^{-1} \sigma$ (with possible singularities) in $\mathbb{R}^{2 n+2}$, up to a constant factor. It follows that $\sigma$ is a length minimizing geodesic "downstairs" (in $\left(\mathbb{R}^{2 n+2} / G, d s^{2}\right)$ ), if and only if $\pi^{-1} \sigma$ is area-minimizing in the class of $G$-invariant $(2 n+1)$-dimensional currents "upstairs" (in $\left(\mathbb{R}^{2 n+2}, d x_{1}^{2}+\cdots+d x_{2 n+2}^{2}\right)$ ), or equivalently, $\pi^{-1} \sigma$ is area-minimizing in $\left(\mathbb{R}^{2 n+2}, d x_{1}^{2}+\cdots+d x_{2 n+2}^{2}\right)$ in general (cf. [13], [2, p. 174, 6.4] and [21]). Furthermore, if a length minimizing geodesic $\sigma$ meets the boundary $\left\{(u, v) \in \mathbb{R}^{2}: u=0, v>0\right\} \cup\left\{(u, v) \in \mathbb{R}^{2}: u>0, v=0\right\}$, it meets the boundary orthogonally by the first variational formula for the arc-length functional, and the corresponding $\pi^{-1} \sigma$ is a regular, embedded and analytic hypersurface in $\mathbb{R}^{2 n+2}$. If $\sigma$ meets the vertex $\{(0,0)\}$, then $\pi^{-1} \sigma$ is singular. Therefore, it suffices to show that any curve in $\mathbb{R}^{2 n+2} / G$, other than the diagonal ray emanating from the origin is not absolutely length minimizing with respect to the metric $d s^{2}=u^{2 n} v^{2 n}\left(d u^{2}+d v^{2}\right)$.

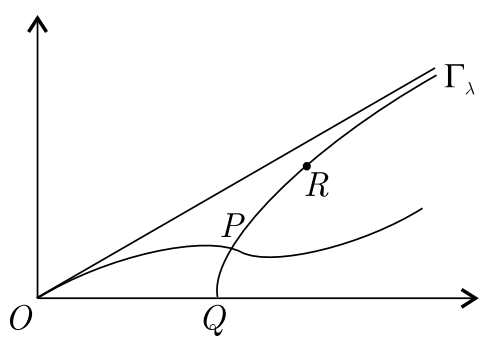


Now let $\Gamma=\left\{\left(u_{0}(t), v_{0}(t)\right)\right\}$ be the geodesic through $(1,0)$ in $\left(\mathbb{R}^{2 n+2} / G, d s^{2}\right)$, and $\Gamma_{\lambda}=$ $\left\{\left(\lambda u_{0}(t), \lambda v_{0}(t)\right)\right\}, \lambda>0$. In [3], a 1-harmonic function was constructed in such a way that the lift of family $\left\{\Gamma_{\lambda}\right\}$ of these homothetic geodesics are level hypersurfaces in $\left(\mathbb{R}^{2 n+2}, d x_{1}^{2}+\cdots+d x_{2 n+2}^{2}\right)$. Hence $\Gamma_{\lambda}$ is absolutely length minimizing in $\left(\mathbb{R}^{2 n+2} / G, d s^{2}\right)$ (cf. also Theorem 2, Remark 2). Now suppose Theorem 3 were not true. Then there would exist a curve $Q P \subset \Gamma_{\lambda}$ transverse to a length minimizing curve $O P$. It follows that the length $l(O P)$ of $O P$ would satisfy $l(O P)=l(Q P)$. Consider the curve $O P R$ where $R$ is on the curve $\Gamma_{\lambda}$, and $l(O P R)=l(Q P R)$. Then the curve $O P R$ would be a geodesic, and hence smooth at $P$. This is a contradiction. Similarly, one can show the remaining case $m \neq n$.

Theorem 4. The cone $C\left(S^{1} \times S^{5}\right)$ over $S^{1} \times S^{5}$ is not absolutely area-minimizing, although it is stable.

Proof. Suppose, on the contrary, that the cone were absolutely area-minimizing. Then consider Lie group $G=S O(2) \times S O(6)$ acting on manifold $\mathbb{R}^{2} \times \mathbb{R}^{6}$ in the standard way. By the previous argument, this would imply the line segment $\overline{O P}$ were length-minimizing in $\left(\mathbb{R}^{8} / G, d s^{2}\right)$, where $d s^{2}=u^{2} v^{6}\left(d u^{2}+d v^{2}\right)$. On the other hand, based on the study of Simoes' thesis [17], [13] and [21], the level curve $\left(u_{\lambda}, v_{\lambda}\right)$ in the $u, v$-plane is absolutely length-minimizing. Argue as before, the curve $O P R$ would be smooth at $P$. This is a contradiction. The stability of the cone follows from Simons' work [18].

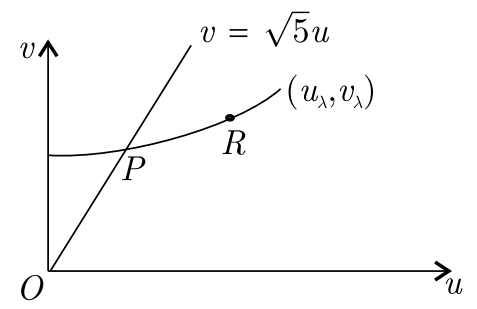

Theorem 5. Any 7-dimensional $S O(2) \times S O(6)$-invariant absolutely area- minimizing integral current in $\mathbb{R}^{8}$ is real analytic.

Proof. By the argument given in the proof of Theorem 3, it suffices to show that any curve in $\mathbb{R}^{2 n+2} / G$, from the origin is not absolutely length minimizing with respect to the metric $d s^{2}=u^{2} v^{6}\left(d u^{2}+d v^{2}\right)$. By Theorem 4, the diagonal ray emanating from the origin is not length minimizing. Similarly, if there were an absolutely length minimizing curve starting from the origin lying above $v=\sqrt{5} u$, then this would lead to an irregularity of a geodesic, a contradiction.

\section{Comparison theorem}

It is known that each level hypersurface of a function of least gradient defined on an open subset $A \subset \mathbb{R}^{n}$ is absolutely area-minimizing in $A$ over $\mathbb{Z}$. It is tempting to ask it if is absolutely area-minimizing in $A$ over $\mathbb{R}$. This motivates our discussion on comparison between real and integral absolute (or homological) minima. In general they are distinct. Examples are given by Almgren [7, 5.11], Federer [7] and Lawson [12]. Furthermore, in the case of 1-dimensional (or co-dimension 1) integral flat chains, Federer [7] has shown that real and integral homological (or absolute) minimizing are the same.

Let $\bar{M}$ be a locally Lipschitz neighborhood retract in $\mathbb{R}^{n}$ (i.e. there exists a locally Lipschitz map which retracts a neighborhood of $\bar{M}$ onto $\bar{M}), M$ be an open subset of $\bar{M}$, and $A$ be an open subset of $\mathbb{R}^{n}$. Using the assumption on vanishing topology, an exhaustion of $M$ by an increasing sequence of compact set $K_{i} \subset M$, we obtain the following: 
Theorem 6. (1) Let $T^{n-1}$ denote a codimension 1 integral absolutely area-minimizing rectifiable current in $M$ with homology group $H_{n-1}(\bar{M})=0$. Then $T^{n-1}$ is absolutely area-minimizing in $M$ if and only if $T^{n-1}$ is absolutely area-minimizing in $A$; and if and only if $T^{n-1}$ is real absolutely area-minimizing in $A$. (2) Let $H_{1}(\bar{M})=0 . T^{1}$ is a homologically area-minimizing rectifiable current of degree 1 of $M$ if and only if $T^{1}$ is real homologically area-minimizing in $M$.

We have the following immediate

Corollary 5. The level hypersurface of a function of least gradient in an open subset $A$ of $\mathbb{R}^{n}$ is absolutely area-minimizing over $\mathbb{R}$.

Corollary 6. Let $N$ be a closed set in $A \subset \mathbb{R}^{n}$ with $H_{n-1}(N)=0$. The graph of any weak solution of the minimal surface equation $\sum_{i=1}^{n} \frac{\partial}{\partial x_{i}}\left(\frac{\frac{\partial f}{\partial x_{i}}}{\sqrt{1+|\nabla f|^{2}}}\right)=0$ on $A-N$ is in fact absolutely area-minimizing in $A \times \mathbb{R} \subset \mathbb{R}^{N+1}$ over $\mathbb{R}$.

Corollary 7. All the examples we find in [21] are absolutely area-minimizing over $\mathbb{R}$.

\section{Acknowledgements}

Research was partially supported by NSF Award No DMS-0508661, OU Presidential International Travel Fellowship, and OU Faculty Enrichment Grant. The author wishes to thank Professors H. Blaine Lawson Jr., and Wu-Yi Hsiang for their interest, and Professor Herbert Federer for his help in Theorem 6 which essentially derives from him. The author also wishes to express his gratitude to the referees and the editors for their comments and suggestions without which the present form of this article would not be possible.

\section{References}

[1] Andreotti A., Vesentini E., Carleman estimate for the Laplace-Beltrami equation on complex manifolds, Inst. Hautes Études Sci. Publ. Math. 25 (1965), 81-130.

[2] Brothers J.E., Invariance of solutions to invariant parametric variational problems, Trans. Amer. Math. Soc. 262 (1980), 159-179.

[3] Bombieri E., de Giorgi E., Giusti E., Minimal cones and the Bernstein problem, Invent. Math. 7 (1969), 243-268.

[4] Federer H., Some theorems on integral currents, Trans. Amer. Math. Soc. 117 (1965) 43-67.

[5] Federer H., Geometric measure theory, Springer, Berlin - Heidelberg - New York, 1969.

[6] Federer H., The singular sets of area minimizing rectifiable currents with codimension one and of area minimizing flat chains modulo two with arbitrary codimension, Bull. Amer. Math. Soc. 76 (1970), 767-771.

[7] Federer H., Real flat chains, cochains and variational problems, Indiana Univ. Math. J. 24 (1974), $351-406$.

[8] Federer H., Fleming W.H., Normal and integral currents, Ann. of Math. (2) 72 (1960), 458-520.

[9] Hsiang W.Y., On the compact homogeneous minimal submanifolds, Proc. Natl. Acad. Sci. USA 56 (1966), 5-6.

[10] Hsiang W.Y., Lawson H.B. Jr., Minimal submanifolds of low cohomogeneity, J. Differential Geom. 5 (1971), $1-38$.

[11] Karp L., Subharmonic functions on real and complex manifolds, Math. Z. 179 (1982), 535-554.

[12] Lawson H.B., The stable homology of a flat torus, Math. Scand. 36 (1975), 49-73.

[13] Lawson H.B., The equivariant Plateau problem and interior regularity, Trans. Amer. Math. Soc. 173 (1972), 231-249.

[14] Lin F.-H., Minimality and stability of minimal hypersurfaces in $R^{N}$, Bull. Austral. Math. Soc. 36 (1987), 209-214. 
[15] Miranda M., Sul minimo dell'integrale del gradiente di una funzione, Ann. Scuola Norm. Sup. Pisa (3) 19 (1965), 626-665.

[16] Miranda M., Comportamento delle successioni convergenti di frontiere minimali, Rend. Sem. Mat. Univ. Padova 39 (1967), 238-257.

[17] Simoes P., A class minimal cones in $\mathbb{R}^{n}, n \geq 8$, that minimizes area, Thesis, Berkeley, 1973.

[18] Simons J., Minimal varieties in riemannian manifolds, Ann. of Math. (2) 88 (1968), 62-105.

[19] Wang S.P., Wei S.W., Bernstein conjecture in hyperbolic geometry, in Seminar on Minimal Submanifolds, Editor E. Bombieri, Ann. of Math. Stud., no. 103, 1983, 339-358.

[20] Wei S.W., Minimality, stability, and Plateau's problem, Thesis, Berkeley, 1980.

[21] Wei S.W., Plateau's problem in symmetric spaces, Nonlinear Anal. 12 (1988), 749-760.

[22] Yau S.T., Some function theoretic properties of complete Riemannian manifolds and their applications to geometry, Indiana Univ. Math. J. 25 (1976), 659-670. 